\title{
Oxidative Stress Is Decreased in Off-pump Versus On-pump Coronary Artery Surgery
}

\author{
Aymelek Gönenç ${ }^{1, *}$, Aysun Hacışevki ${ }^{1}$, Beyhan Bakkaloğlu², Aylin Soyağır ${ }^{2}$, \\ Meral Torun ${ }^{1}$, Haldun Karagöz ${ }^{2}$ and Bolkan Şimşek ${ }^{1}$ \\ ${ }^{1}$ Department of Biochemistry, Faculty of Pharmacy, Gazi University, Ankara, Turkey \\ ${ }^{2}$ Department of Cardiac Surgery, Güven Hospital, Ankara, Turkey \\ Received 9 December 2005, Accepted 21 March 2006
}

Oxidative stress occurs in patients undergoing coronary artery bypass operation. The aim of this study was to investigate the difference in oxidative stress in off-pump versus on-pump coronary artery bypass surgery. In the present study, in serial blood samples, plasma malondialdehyde (MDA) as index of lipid peroxidation, red blood cells glutathione peroxidase (GPx) and superoxide dismutase (SOD) were measured to compare the extent of oxidative stress in 30 patients undergoing OPCAB (off-pump coronary artery bypass grafting), 12 patients undergoing CABG (on-pump coronary artery bypass grafting) and 18 healthy controls. In CABG group, MDA levels increased significantly from $2.87 \pm 0.62 \mathrm{nmol} / \mathrm{mL}$ before anesthesia and $2.87 \pm 0.65 \mathrm{nmol} / \mathrm{mL}$ after anesthesia to $3.05 \pm 0.66$ $\mathrm{nmol} / \mathrm{mL}$ after ischemia $(\boldsymbol{p}<\mathbf{0 . 0 5})$. Similarly, SOD levels also elevated significantly from $661.58 \pm 78.70 \mathrm{U} / \mathrm{g} \mathrm{Hb}$ before anesthesia and $659.42 \pm 81.21 \mathrm{U} / \mathrm{g} \mathrm{Hb}$ anesthesia induction to $678.08 \pm 75.80 \mathrm{U} / \mathrm{g} \mathrm{Hb}$ after ischemia $(p<0.01$, $p<0.01$, respectively). In OPCAB group, only SOD levels increased from $581.73 \pm 86.24 \mathrm{U} / \mathrm{g}$ Hb anesthesia induction to $590.90 \pm 88.90 \mathrm{U} / \mathrm{g} \mathrm{Hb}$ after reperfusion $(p<0.05)$. Glutathione peroxidase levels were not changed according to blood collection times in both of CABG group or OPCAB group $(p>0.05)$. Our results show that only mild signs of oxidative stress is found after reperfusion in OPCAB operation compared with CABG operation.

\footnotetext{
Abbrevations: MDA: malondialdehyde; GPx: glutathione peroxidase; SOD: superoxide dismutase; OPCAB: off-pump coronary artery bypass grafting; CABG: on-pump coronary artery bypass grafting; TEP: 1,1,3,3-tetraethoxypropane; BHT: butylated hydroxytoluene; Trizma base: tris [hydroxymethyl]aminomethane; TBA: 2-thiobarbituric acid; NBT: nitroblau-tetrazolium chlorid
}

\footnotetext{
*To whom correspondence should be addressed. Tel: 90-312-202 31 52/1213; Fax: 90-312-223 5018

E-mail: aymelek@gazi.edu.tr
}

Further studies are needed in order to confirm this hypothesis.

Keywords: Coronary artery bypass, Glutathione peroxidase, Malondialdehyde, Oxidative stress, Superoxide dismutase

\section{Introduction}

Myocardial ischemia-reperfusion represents a clinically relevant problem associated with thrombolysis, angioplasty and coronary bypass surgery. Several studies have proposed the essential role of reactive oxygen species in the pathogenesis of myocardial ischemia-reperfusion injury (Grech et al, 1996; Kato et al., 1998). Reactive oxygen species including superoxide radical, hydroxyl radical and hydrogen peroxide have been shown to increase during reperfusion of the heart following ischemia (Kato et al, 1998). Systemic increase of hydrogen peroxide and lipid peroxidation products has been demonstrated to occur during CABG operation (Kharazmi et al., 1989). The circulating blood to non-physiological surfaces in CABG can be considered as another potential source of oxidative stress. It has been demonstrated that extracorporeal circulation in dialysis patients elevated free radical production (Eiselt et al., 2001). OPCAB operation does not involve extracorporeal pumping and circulation of blood.

Peroxidation of membrane phospholipid polyunsaturated fatty acids is considered as a major mechanism of the damage occuring on reperfusion of the myocardium after a prolonged period of ischemia (Ytrehus and Hegstad, 1991). An increase in the formation of reactive oxygen species during ischemiareperfusion and the adverse effects of oxyradicals on myocardium have now been well established by both direct and indirect measurements. Malondialdehyde is one of the small molecular-mass compounds resulting from fragmentation 
of polyunsaturated fatty acids undergoing ROS attack which is a fact that qualifies this aldehyde as a marker of lipid peroxidation.

In most mammalian species, SOD and GPx appear to be the most active antioxidant enzymes in the myocardium. SOD catalyses the dismutation reaction of the toxic superoxide radical to molecular oxygen and hydrogen peroxide and thus forms a crucial part of the cellular antioxidant defence mechanism. The role of SOD in myocardial ischemiareperfusion injury has been the subject of much controversy (Arduini et al., 1988; Turrens et al., 1992; Chen et al., 2000). GPx catalyzes the peroxidation of hydrogen peroxide in the presence of reduced glutathione (GSH) to form water and oxidized glutathione (GSSG). The oxidized glutathione recycles back to give reduced glutathione by glutathione reductase, which requires NADPH. Thus, GPx plays a significant role as hydrogen peroxide scavenger in the heart. An increasing number of investigators have demonstrated the importance of glutathione peroxidase in protecting myocardium from ischemia-reperfusion (Ferrari et al. 1985; Arduini et al., 1988; Carlucci et al., 2002).

The present study was designed to investigate the difference in oxidative stress in OPCAB versus CABG operations before anesthesia, after anesthesia and ischemia and reperfusion times. Hence, all patients in the study were operated under identical conditions, and the only variable was the use of different bypass operation methods.

\section{Materials and Methods}

Materials. 1,1,3,3-tetraethoxypropane (TEP), butylated hydroxytoluene (BHT), o-phosphoric acid, potassium ferricyanide, cupric chloride dihydrate, xanthine sodium salt, xanthine oxidase, trizma base (tris[hydroxymethyl] aminomethane, bovine serum albumin and glutathione were obtained from Sigma (St. Louis, MO). Ethanol, HPLC-grade methanol, 2-thiobarbituric acid (TBA), potassium dihydrogenphosphate, dinatrium hydrogenphosphate, potassium cyanide, natrium azid, sodium carbonate anhydrous, sodium hydrogen carbonate, nitroblau-tetrazolium chlorid (NBT) were purchased from Merck (Darmstadt). Superoxide dismutase and glutathione reductase were obtained from Roche (Mannheim). All other chemicals used were of analytical grade.

\section{Patients and controls}

Forty-two patients (33 male and 9 female, mean age of $64.6 \pm 1.2$ $\mathrm{yr})$ as a total were included in this study. Patients were randomly assigned to OPCAB or CABG. Thirty patients (24 male and 6 female) were operated on by OPCAB technique. Twelve patients ( 9 male and 3 female) were operated on by using the CABG tecnique. Patients who used antioxidants such as captopril and allopurinol were excluded from the study. Patients who received blood transfusion or blood products during the operation were also excluded, since the antioxidant properties of such products are not as yet established. None of the patients were taking vitamins or dietary supplements with established antioxidant properties before the study. The study was approved by Gazi University Medical Ethics Committee, and written informed consent was obtained from all participants. The type II DM was assessed by World Health Organization criteria in diabetic coronary artery patients. Control group were included 18 healthy subjects (16 male and 2 female, mean age of $69.7 \pm 1.5 \mathrm{yr}$ ). None of the controls had a history of cerebrovascular or ischemic heart disease.

\section{Anesthetic technique}

Anesthesic technique was standardized for all patients and consisted of balanced anesthesia. All patients were premedicated with diazepam $5 \mathrm{mg}$ before the night and with midazolam $0.07 \mathrm{mg} /$ $\mathrm{kg}$ i.m. 30 minute before the surgery. Patients underwent anesthesia induction with midazolam $(0.15 \mathrm{mg} / \mathrm{kg})$, fentanyl $(4 \mu \mathrm{g} / \mathrm{kg})$ and propofol (1-2 mg/kg). Pancuronium or vecuronium was used as a muscle relaxant $(0.1-0.15 \mathrm{mg} / \mathrm{kg})$. After tracheal intubation, anesthesia was maintained with a $50 \%$ air/oxygen mixture with isoflurane in a concentration of $0.5-1 \%$. Additional fentanyl and propofol were applied when necessary.

\section{Surgical procedure}

OPCAB operation. The operation was performed through a median sternotomy. After harvesting the bypass conduits, heparin was given at a dose of $100 \mathrm{IU} / \mathrm{kg}$ to achieve a target activated clotting time of 250 to 300 seconds. Beta blocker drugs were used to decrease heart rate during the anastomosis. Any myocardial stabilization device were not used except cotton sutures. During the anastomosis coronary flow was occluded by bulldog clamps. The proximal anastomosis was performed using a site clamp on the aorta or on the internal mammary artery. All grafts were arterial (IMA or radial artery).

CABG operation. The operation was performed through median sternotomy. After harvesting the bypass conduits, heparin was given at a dose of $400 \mathrm{IU} / \mathrm{kg}$ to achieve a target activated clotting time of 450 seconds or above. Cardiopulmonary bypass operation was instituted using ascending aortic cannulation and two-stage venous cannulation in the right atrium. The extracorporeal circuit consisted of a membran oxygenator and a roller pump primed with crystalloid solution. CABG was managed according to the alphastat principle, moderate hypotermia, and the CABG flow rate was maintained at $2.4 \mathrm{l} / \mathrm{min}$ per $\mathrm{m}^{2}$. Myocardial protection was achieved with cold potassium cardioplegia (Plegisol; Abbot Inc.Chicago IL), and warm blood cardioplegia before removing the aortic crossclamp. The cardioplegia was given retrogradely except the first two-thirds of cristalloid cold cardioplegia, was given anterogradely. All distal and proximal anastomosis were completed before the aortic cross-clamp was removed. At the end of CABG heparin was neutralised by protamine chloride until the activated clotting time was less then 180 seconds. In the CABG group hematocrit was kept more than $20 \%$ during $\mathrm{CPB}$, whereas in the OPCAB group hematocrit was kept more than $25 \%$.

\section{Sample collection and analyses}

Blood samples were collected at different time points according to the protocol described in Table 1. In all patients the first samples were obtained from the venous line, while other times blood 
Table 1. Blood sampling protocol for comparative measurements of oxidative stress markers in CABG and OPCAB patients

\begin{tabular}{clc}
\hline Time & \multicolumn{1}{c}{ Group CABG } & Group OPCAB \\
\hline $\mathrm{t} 1$ & Before anesthesia & Before anesthesia \\
$\mathrm{t} 2$ & $10 \mathrm{~min}$ after anesthesia induction & 10 min after anesthesia induction \\
$\mathrm{t} 3$ & $10 \mathrm{~min}$ after cross-clamp (ischemia) & - \\
$\mathrm{t} 4$ & 10 min after cessation of CPB (reperfusion) & 10 min after cessation of CPB (reperfusion) \\
\hline
\end{tabular}

samples were obtained from arterial line. The blood was drawn into plastic syringes and plasma was separated by centrifugation and kept in plastic tubes at $-70^{\circ} \mathrm{C}$ until analysis. Samples were obtained before anesthesia ( $\mathrm{t} 1$ ), before operation ( $\mathrm{t} 2), 10$ minutes after start of CABG operation (ischemic period) (t3), 10 minutes after cessation of $\mathrm{CPB}$ (reperfusion time) (t4) in CABG group. In the OPCAB group blood collection were similar to CABG group except ischemic period ( $\mathrm{t} 3$ ). Samples were collected before anesthesia ( $\mathrm{t} 1)$, before operation ( $\mathrm{t} 2)$ and 10 minutes after cessation of CPB (reperfusion time) (t4) in OPCAB group.

MDA assay. The EDTA-anticoagulated samples were centrifuged at $3,000 \mathrm{rpm}$ for $5 \mathrm{~min}$ at $4^{\circ} \mathrm{C}$. The plasma was aliquoted. The thiobarbituric acid reaction was carried out by mixing $50 \mu \mathrm{L}$ of $0.05 \%$ (w/v) BHT solution, $400 \mu \mathrm{L}$ of $0.44 \mathrm{M} \mathrm{H}_{3} \mathrm{PO}_{4}$ solution, 100 $\mu \mathrm{L}$ of $42 \mathrm{mM}$ TBA solution and $50 \mu \mathrm{L}$ sample or TEP standard $(0-$ $5 \mu \mathrm{M})$. Sample tubes were heated for $1 \mathrm{~h}$ on a $100^{\circ} \mathrm{C}$. n-butanol $(250 \mu \mathrm{L})$ was added to each tube for extraction of the MDA-TBA complex. Samples were analyzed for malondialdehyde by HPLC with fluorometric detection using an excitation wavelength of 532 $\mathrm{nm}$ and emission wavelength of $553 \mathrm{~nm}$ (Hewlett-Packard model 1046A programmable fluorescence detector) (Agarwal and Chase, 2002). A Lichrospher $100 \mathrm{RP}-18 \mathrm{e}$ column ( $5 \mu \mathrm{m}, 4 \mathrm{mmx} 250 \mathrm{~mm})$ was used. The mobile phase contained $40 \%$ methanol and $60 \%$ phosphate buffer at $\mathrm{pH} 6.8$. A flow rate of $1 \mathrm{ml} / \mathrm{min}$ was used.

Assay of GPx activity. GPx catalyzes the oxidation of reduced glutathione to oxidized glutathione by hydrogen peroxide. The formation rate of oxidized form was measured by means of glutathione reductase reaction. Glutathione reductase catalyzes the reduction of oxidized form by NADPH as cofactor. The enzymatic activity was spectrophotometrically estimated using the accompanying oxidation of NADPH. The erythrocytes were lysed with ice-cold water and the hemolysate was diluted. The method of Pleban et al. was used. The decrease of absorbance was measured against the blank sample at $340 \mathrm{~nm}$ using a Beckman Du 650 spectrophotometer.

Assay of Cu,Zn-SOD Activity. The assay for SOD was based on the ability of the enzyme to accelerate the dismutation of superoxide produced by the the xanthine/xanthine oxidase system. Red cells were washed 3 times with $0.9 \% \mathrm{NaCl}$. The erythrocytes were lysed with ice-cold water. The hemoglobin was then removed by adding a chloroform/ethanol mixture. The mixture was centrifuged for $45 \mathrm{~min}$ at 12,200 rpm. The supernatant fluid was diluted for the enzymatic assay. The method of Sun et al. was used. The reduction of nitroblue tetrazolium was measured at $560 \mathrm{~nm}$ using a Beckman Du 650 spectrophotometer. The inhibition of the SOD reaction was evaluated and calibrated with bovine erythrocytes as a standard.
Measurement of other parameters. Serum total cholesterol, HDLcholesterol, triglycerides, fasting blood glucose, ALT, AST, albumin and total bilirubin were measured spectrophotometrically on the Hitachi 912 autoanalyzer by using a commercial kit (Roche, Mannheim, Germany). Concentration of serum LDL-cholesterol was calculated by the standard Friedewald formula.

\section{Data analysis}

All data are expressed as mean value \pm standard deviation of the mean. Statistical analysis of the results was performed using SPSS for Windows version 10.0. Student's t-test was used to compare the results between the patients and healthy controls. One-way analysis of variance was performed in repeated measures. Pearson correlation coefficients were calculated in order to test the relationship between measured parameters. All $\mathrm{p}$ values $<0.05$ were considered to be significant.

\section{Results}

No patient received inotropic support during the operation and none developed any significant complication over $24 \mathrm{~h}$ of observation, i.e. stroke, arrhythmia, renal failure, pulmonary insufficiency, infection or excessive bleeding procedures. During the study period there was no mortality in patients.

The general characteristics and measured parameters of the study population before operation are shown in Table 2 . The mean fasting blood glucose, triglycerides, total bilirubin levels in total bypass patients were higher than that of healthy controls $(p<0.05, p<0.01$ and $p<0.05$, respectively), but HDL-cholesterol and AST levels were lower than those of control group ( $p<0.01, p<0.01$, respectively). There was no differences between the total patients and controls according to LDL-cholesterol, ALT, albumin and hemoglobin levels $(p>0.05)$. MDA levels of the total patients were higher approximately $30 \%$ than healthy controls $(p<0.05)$ (Table 2$)$. There was no difference in glutathione peroxidase levels between the total patients and controls $(p>0.05)$ (Table 2). The mean erythrocyte SOD levels in total patients were lower approximately $23 \%$ compared with control group $(p<0.01)$ (Table 2).

The mean MDA level was elevated in the CABG group from $2.87 \pm 0.62 \mathrm{nmol} / \mathrm{mL}$ preoperatively to $3.05 \pm 0.66 \mathrm{nmol} /$ $\mathrm{mL}$ during ischemic period $(p<0.05)$ (Figure 1a). Preoperative MDA levels were not changed after anesthesia induction $(2.87 \pm 0.65 \mathrm{nmol} / \mathrm{mL})(p>0.05)$. The MDA levels increased during ischemic period compared with after anesthesia 
Table 2. Comparison of measured blood parameters in total bypass patients and controls $(\mathrm{X} \pm \mathrm{S}$.E.)

\begin{tabular}{lcc}
\hline & Total bypass patients $(\mathrm{N}=42)$ & Healthy controls $(\mathrm{N}=18)$ \\
\hline Age (years) & $64.6 \pm 1.2 \mathrm{yr}$ & $69.7 \pm 1.5 \mathrm{yr}$ \\
Sex $(\mathrm{M} / \mathrm{F})$ & $33 / 9$ & $16 / 2$ \\
MDA (nmol/mL) & $2.6 \pm 0.1^{\mathrm{a}}$ & $21.9 \pm 0.3$ \\
GPx ((U/g Hb) & $20.8 \pm 0.4$ & $743.4 \pm 31.8$ \\
SOD (U/g Hb) & $605.3 \pm 14.1^{\mathrm{b}}$ & $175.3 \pm 3.4$ \\
Total cholesterol (mg/dL) & $193.3 \pm 8.2$ & $51.0 \pm 1.6$ \\
HDL-cholesterol (mg/dL) & $42.6 \pm 1.7^{\mathrm{b}}$ & $98.6 \pm 2.9$ \\
LDL-cholesterol (mg/dL) & $110.3 \pm 5.7$ & $102.4 \pm 7.6$ \\
Triglycerides (mg/dL) & $237.7 \pm 26.5^{\mathrm{b}}$ & $101.7 \pm 3.7$ \\
Fasting blood glucose (mg/dL) & $135.4 \pm 10.5^{\mathrm{a}}$ & $18.9 \pm 0.9$ \\
ALT (U/L) & $23.3 \pm 1.7$ & $26.39 \pm 3.0$ \\
AST (U/L) & $18.8 \pm 0.8^{\mathrm{b}}$ & $4.2 \pm 4.7 \times 10^{-2}$ \\
Albumin (mg/dL) & $4.2 \pm 5.5 \times 10^{-2}$ & $14.08 \pm 0.28$ \\
Haemoglobin (g/dL) & $14.21 \pm 0.24$ & $1.0 \pm 4.9 \times 10^{-2}$ \\
Total bilirubin (mg/dL) & $1.0 \pm 0.2^{\mathrm{a}}$ & \\
\hline
\end{tabular}

${ }^{\mathrm{a}}$ significant difference from control group $(p<0.05)$

bsignificant difference from control group $(p<0.01)$.

induction $(p<0.05)$. The increase in ischemic period was remained until reperfusion time, after the mean MDA level decreased to $2.97 \pm 0.51 \mathrm{nmol} / \mathrm{mL}(p>0.05)$. GPx levels were similar according to blood collection times in CABG group. The GPx values at specific time-points were $\mathrm{t} 1=19.58 \pm$ $2.23, \mathrm{t} 2=19.33 \pm 2.06, \mathrm{t} 3=19.50 \pm 2.28$ and $\mathrm{t} 4=19.75 \pm$ $1.91 \mathrm{U} / \mathrm{g} \mathrm{Hb}$ (Fig. 1b). The mean SOD levels increased significantly after ischemia compared with preoperative values. This increase was from $661.58 \pm 78.70 \mathrm{U} / \mathrm{g} \mathrm{Hb}$ to 678.08 $\pm 75.80 \mathrm{U} / \mathrm{g} \mathrm{Hb}(p<0.01)$ (Figure 1c). The SOD levels after anesthesia induction, $659.42 \pm 81.21 \mathrm{U} / \mathrm{g} \mathrm{Hb}$, were similar to preoperative SOD levels $(p>0.05)$. The SOD levels elevated significantly during ischemic period compared with anesthesia induction $(p<0.01)$. The mean SOD level during ischemic period diminished to $667.83 \pm 78.76 \mathrm{U} / \mathrm{g} \mathrm{Hb}$ after reperfusion $(p>0.05)$.

In OPCAB group, MDA levels were not different in before anesthesia, after anesthesia and reperfusion groups. The MDA levels were $\mathrm{t} 1=2.49 \pm 0.65, \mathrm{t} 2=2.50 \pm 0.65$ and $\mathrm{t} 4=2.52 \pm$ $0.67(p>0.05)$ (Figure 1a). GPx levels were not different at blood collection times in this group. GPx levels in OPCAB group were $\mathrm{t} 1=21.27 \pm 3.05, \mathrm{t} 2=21.10 \pm 3.46$ and $\mathrm{t} 4=$ $21.33 \pm 3.29(p>0.05)$ (Figure 1b). The mean SOD level was increased in the OPCAB group from $581.73 \pm 86.24 \mathrm{U} / \mathrm{g} \mathrm{Hb}$ after anesthesia induction to $590.90 \pm 88.90 \mathrm{U} / \mathrm{g} \mathrm{Hb}$ after reperfusion time. The SOD levels after anesthesia induction were similar to preoperative SOD levels $(582.83 \pm 87.53 \mathrm{U} / \mathrm{g}$ $\mathrm{Hb})(p>0.05)$ (Fig. 1c). As a result we observed that CABG operation had more effects on coronary artery patients compared with OPCAB operation.

Using bivariate correlation analysis of the measured parameters, no significant correlation was found between oxidative stress parameters $(p>0.05)$.

\section{Discussion}

Ischemia-reperfusion injury to cardiac myocytes involves membrane damage mediated by oxygen free radicals (McCord 1988; Lucas and Szweda, 1998). At the cellular level, the excessive production of oxygen free radicals causes lipid peroxidation, protein denaturation, enzyme inactivation, carbohydrate breakdown and, finally tissue injury (Eiselt et $a l ., 2001)$. In CPB, the heart undergoes ischemia-reperfusion, myocyte damage occurs due to ATP and defense mechanisms depletion against free radical injury (Menasche and Piwnica, 1989; Carlucci et al., 2002). In this study, we tried to demonstrate how oxidative stress parameters differ in ischemiareperfusion between the two groups undergoing off- and onpump CPB.

Some previous studies have demonstrated that reactive oxygen species are generated during $\mathrm{CABG}$, lipid peroxidation occurs during the operation and pretreatment with antioxidants before CABG minimizes lipid peroxidation (Arduini et al., 1988; Carlucci et al., 2002). Starkopf et al. have reported that serum thiobarbituric acid reactive substances (TBARS) level elevated during $\mathrm{CABG}$ operation compared to before operation. Paradies et al. have found a significant increase in MDA production in both ischemia and reperfusion times (Paradies et al., 1999). In the present study, we demonstrate a significant increase in plasma MDA levels in CABG group during ischemic period compared with before operation and anesthesia induction. At the end of reperfusion phase, MDA concentrations still remained elevated. Activated neutrophils, xanthine oxidase of endotelial cells, and damaged heart mitocondria could be a potential sources of free radical production during $\mathrm{CABG}$ operation. Extracorporeal circulation in $\mathrm{CABG}$ operation, by increasing contact of blood with 


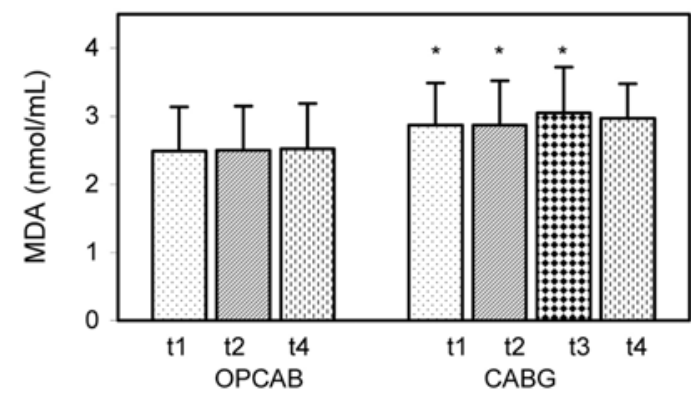

*significant difference between $\mathrm{t} 1$ and $\mathrm{t} 3$, $\mathrm{t} 2$ and $t 3$ in $C A B G$ group $(p<0.05, p<0.05$, respectively).
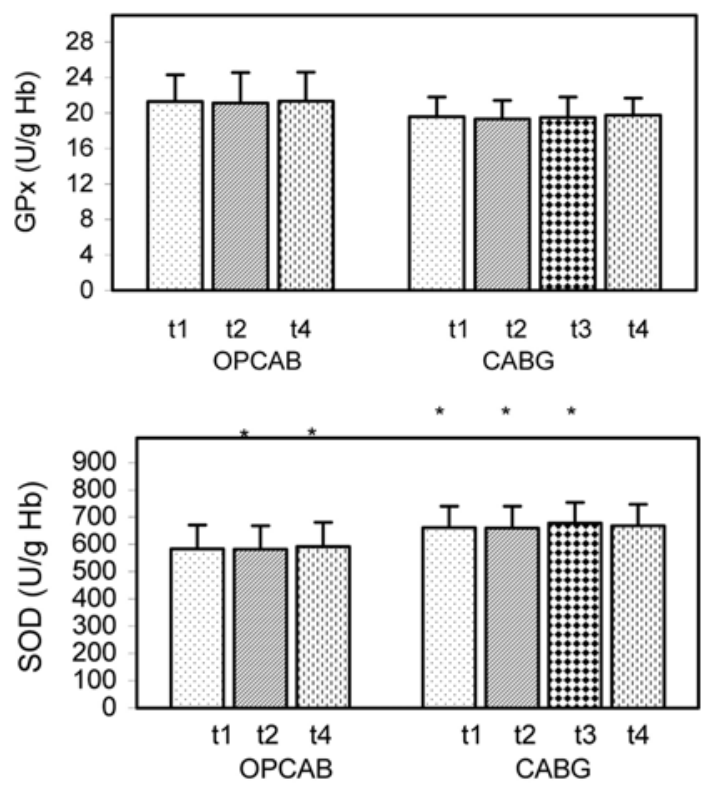

*significant difference between $\mathrm{t} 2$ and $\mathrm{t} 4$ in OPCAB group $(\mathrm{p}<0.05)$; $\mathrm{t} 1$ and $\mathrm{t} 3, \mathrm{t} 2$ and $\mathrm{t} 3$ in CABG group ( $p<0.01, p<0.01$, respectively).

Fig. 1. Comparison of MDA (a), GPx (b) and SOD (c) levels at different time points in OPCAB and CABG method.

foreign substances, may also induce systemic inflammatory responses. On-pump procedures are associated with higher degrees of oxidative stress than off-pump coronary surgery (Matata et al.,2000; Gerritsen et al., 2001; Cavalca et al., 2006). In a prospective study comparing OPCAB with CABG, OPCAB patients displayed lower levels of protein carbonylation and nitration, lipid hydroperoxides (Matata et al., 2000). Similarly, Gerritsen et al. showed that OPCAB was associated with lower levels of urinary xanthine, hypoxanthine and malondialdehyde after operation. In this study, elevated MDA levels were found during ischemic period in CABG group compared to OPCAB group. These results are in accordance with previous studies (Matata et al., 2000; Gerritsen et al., 2001). This significant difference in MDA levels may be due to the longer duration of ischemia, and to the global rather than regional ischemia induced during
CABG compared to OPCAB surgery.

The levels of intermediate reduction products of oxygen metabolism are controlled by various cellular defence systems consisting of enzymatic (SOD, GPx, glutathione reductase and catalase) and non-enzymatic (vitamin $\mathrm{E}$ and glutathione) scavenger components. Oxidative stress is the result of imbalance between the formation of reactive oxygen species and local antioxidant defences. Changes in antioxidant enzyme activity in erythrocytes have been used to document oxidative stress. SOD functions in the cell against superoxide radicals. Conflicting results regarding the protective effects of SOD against ischemia-reperfusion injury have been reported (Arduini et al., 1988; Turrens et al., 1992; Chen et al., 2000). An earlier study has shown that myocardial mitochondrial Mn-SOD activity was diminished after ischemia (Turrens et al., 1992). However, another study has reported that $\mathrm{Cu}, \mathrm{Zn}-$ SOD didn't change while glutathione peroxidase and reductase activities were increased (Arduini et al., 1988). Chen et al.and Luyten et al. showed that overexpression of Cu,ZnSOD in coronary vascular cells rendered the heart more resistant to ischemia/reperfusion injury. In the present study, increased SOD levels were found during ischemia-reperfusion in both $\mathrm{CABG}$ and OPCAB groups. The possible explanation for this elevation is that the rise in SOD activity could be compensatory mechanism of the body to prevent tissue damage. Our findings illustrate that SOD is a strong first line of defence against superoxide radicals. Moreover, SOD is enzymatic antioxidant which is not consumed during their detoxification activities. Increased SOD levels are more significant in CABG group than OPCAB group. This can be explained by the fact that contact of blood with polymers of the CPB circuit release cytokines and activates neutrophils ( $\mathrm{Li}$ et al., 2004). Increased free radical production has been demonstrated in patients undergoing dialysis (Eiselt et al, 2001).

Some studies confirm the role of glutathione peroxidase in cardioprotection against ischemia-reperfusion (Arduini et al., 1988; Carlucci et al., 2002). Carlucci et al. found an imbalance in plasma GPx, the cellular GSH/GSSG ratio and plasma levels of nitrate/nitrite during ischemia. Their data indicate that GPx levels diminish after ischemia and then increase to before cross-clamping levels. Similary, Van Jaarsveld et al. observed a decrease in GPx activity. In contrast, some studies have shown that there is a significant elevation of GPx levels during operation (Arduini et al., 1988; Luyten et al., 2005). Few studies could not find any significant change in GPx activities (Ferrari et al., 1985; Inal et al., 1999). In rabbit heart, GPx and reductase activities were found to be unaltered after ischemia (Ferrari et al., 1985). In this study, we found that the activity of the enzymatic antioxidant GPx levels did not change during ischemiareperfusion in both groups. Our results are in accordance with those reported by Ferrari et al. We found only increased SOD levels, but not GPx levels, in CABG patients during ischemia and $\mathrm{OPCAB}$ patients at reperfusion time. It may be associated with elevated lipid peroxidation. The SOD activity seems to 
be more susceptible against to reactive oxygen species compared to GPx activity.

\section{Conclusions}

Our results indicate that patients undergoing OPCAB had only mild signs of oxidative stress compared with patients submitted to CABG. MDA and SOD levels increased in $\mathrm{CABG}$ operation during ischemia. We conclude that OPCAB surgery decreases impairment of oxidant-antioxidant balance. A larger study with a sample size capable to detect differences in clinical outcome is now warranted.

Acknowlegments This work is supported by Gazi University Research Foundation (Project no 02/2001-18).

\section{References}

Agarwal, R. and Chase, S. D. (2002) Rapid, fluorimetric-liquid chromatographic determination of malondialdehyde in biological samples. J. Chromatogr. B 775, 121-126.

Arduini, A., Mezzetti, A., Porreca, E., Lapenna, D., Dejulia, J., Marzio, L., Polidoro, G. and Cuccurullo, F. (1988) Effect of ischemia and reperfusion on antioxidant enzymes and mitocondrial inner membrane proteins in perfused rat heart. Biochim. Biophys. Acta 970, 113-121.

Carlucci, F., Tabucchi, A., Biagioli, B., Simeone, F., Scoletta, S., Rosi, F. and Marinello, E. (2002) Cardiac surgery: myocardial energy balance, antioxidant status and endothelial function after ischemia-reperfusion. Biomed. Pharmacother. 56, 483-491.

Chen, Z., Oberley, T. D., Ho, Y. S., Chua, C. C., Siu, B., Hamdy, R. C., Epstein, C. J. and Chua, B. H. (2000) Overexpression of $\mathrm{CuZnSOD}$ in coronary vascular cells attenuates myocardial ischemia/reperfusion injury. Free Radic. Biol. Med. 29, 589596.

Cavalca, V., Sisillo, E., Veglia, F., Tremoli, E., Cighetti, G., Salvi, L., Sola, A., Mussoni, L., Biblioli, P., Folco, G., Sala, A. and Parolari, A. (2006) Isoprostanes and oxidative stress in offpump and on-pump coronary bypass surgery. Ann. Thorac. Surg. 81, 562-567.

Eiselt, J., Racek, J. and Opatrny, K. J. (2001) Paired filtration dialysis and free radicals. Cas Lek Cesk 140, 238.

Ferrari, R., Ceconi, C., Curello, S., Guarnieri, C., Calderera, C. M., Albertini, A. and Visioli, O. (1985) Oxygen-mediated myocardial damage during ischemia and reperfusion: role of the cellular defences against oxygen toxicity. J. Mol. Cell. Cardiol. 17, 937-945.

Gerritsen, W. B., Boven, W. J., Driessen, A. H., Haas, J. F. and Aarts, L. P. (2001) Off-pump versus on pump coronary artery bypass grafting: oxidative stress and renal function. Eur. $J$. Cardio-Thorac. 20, 923-929.

Grech, E. D., Dodd, N. J., Jackson, M. J., Morrison, W. L., Fragher, E. B. and Ramsdale, D. R. (1996) Evidence for free radical generation after primary percutaneous transluminal coronary angioplasty recanalization in acute myocardial infarction. Am. J. Cardiol. 77, 122-127.

Inal, M., Alatas, O., Kanbak, G., Akyüz, F. and Sevin, B. (1999) Changes of antioxidant enzyme activities during cardiopulmonary bypass. J. Cardiovasc. Surg. 40, 373-376.

Kato, K., Shao, Q., Elimban, V., Lukas, A. and Dhalla, N. S. (1998) Mechanism of depression in cardiac sarcolemmal $\mathrm{Na}^{+}-$ $\mathrm{K}^{+}$ATPase by hypochlorous acid. Am. J. Physiol. 275, 826831.

Kharazmi, A., Andersen, L. W., Baek, L., Valerius, N. H., Laub, M. and Rasmussen, J. P. (1989) Endotoxemia and enhanced generation of oxygen radicals by neutrophils from patients undergoing cardiopulmonary bypass. J. Thorac. Cardiovasc. Surg. 98, 381-385.

Li, J. M. and Shah, A. M. (2004) Endothelial cell superoxide generation: regulation and relevance for cardiovascular pathophysiology. Am. J. Physiol. Regul. Integr. Comp. Physiol. 287, 1014-1030.

Lucas, D. T. and Szweda, L. I. (1998) Cardiac reperfusion injury:aging, lipid peroxidation and mitocondrial dysfunction. Proc. Natl. Acad. Sci. USA 95, 510-514.

Luyten, C. R., Van Overveld, F. J., De Backer, L. A., Sadowska, A. M., Rodrigus, I. E., De Hert, S. G. and De Backer, W. A. (2005) Antioxidant defence during cardiopulmonary bypass surgery. Eur. J. Cardio-thorac. Surg. 27, 611- 616.

Matata, B. M., Sosnowski, A. W. and Galinanes, M. (2000) Off pump bypass graft operation significantly reduces oxidative stress and inflammation. Ann. Thorac. Surg. 69, 785-791.

McCord, J. M. (1988) Free radicals and myocardial ischemia: overview and Outlook. Free Radic. Biol. Med. 4, 9-14.

Menasche, P. and Piwnica, A. (1989) Free radicals and myocardial protection: a surgical viewpoint. Ann. Thorac. Surg. 47, 939.

Paradies, G., Petrosillo, G., Pistolese, M., Di Verosa, N., Serena, D. and Ruggiero, F. M. (1999) Lipid peroxidation and alterations to oxidative metabolism in mitochondria isolated from heart subjected to ischemia and reperfusion. Free Radic. Biol. Med. 27, 42-50.

Pleban, P. A., Munyani, A. and Beachum, J. (1982) Determination of selenium concentration and glutathione peroxidase activity in plasma and erythrocytes. Clin. Chem. 28, 311-316.

Sun, Y. I., Oberley, W. and Li, Y. (1988) Simple method for clinical assay of superoxide dismutase. Clin. Chem. 34, 497500.

Starkopf, J., Tamme, K., Zilmer, M., Talvik, R. and Samarutel, J. (1997) The evidence of oxidative stress in cardiac surgery and septic patients: a comparative study. Clin. Chim. Acta 262, 7788.

Turrens, J. F., Thornton, J., Barnard, M. L., Synder, S., Liu, G. And Downey, J. M. (1992) Protection from reperfusion injury by preconditioning hearts does not involve increased antioxidant defences. Am. J. Physiol. 262, H585-H589.

Van Jaarsveld, H., Groenewald, A. J., Potgieter, G. M., Barnard, S. P., Vermaak, W. J. and Barnard, H. C. (1988) Effect of normothermic ischemic cardiac arrest and of reperfusion on the free oxygen radical scavenger enzymes and xanthine oxidase. Enzyme 39, 8-16.

Ytrehus, K. and Hegstad, A. C. (1991) Lipid peroxidation and membrane damage of heart. Acta Physiol Scand 599, 81-91. 